\title{
Solar wind modulation of the Martian ionosphere observed by Mars Global Surveyor
}

\author{
J.-S. Wang ${ }^{1,2}$ and E. Nielsen ${ }^{1}$ \\ ${ }^{1}$ Max-Planck-Institut für Aeronomie, 37191 Katlenburg-Lindau, Germany \\ ${ }^{2}$ School of Earth and Space Sciences, Peking University, Beijing 100871, China \\ Received: 8 August 2003 - Accepted: 18 February 2004 - Published: 14 June 2004
}

\begin{abstract}
Electron density profiles in the Martian ionosphere observed by the radio occultation experiment on board Mars Global Surveyor have been analyzed to determine if the densities are influenced by the solar wind. Evidence is presented that the altitude of the maximum ionospheric electron density shows a positive correlation to the energetic proton flux in the solar wind. The solar wind modulation of the Martian ionosphere can be attributed to heating of the neutral atmosphere by the solar wind energetic proton precipitation. The modulation is observed to be most prominent at high solar zenith angles. It is argued that this is consistent with the proposed modulation mechanism.
\end{abstract}

Key words. Ionosphere (Planetary ionosphere; Ionospheric disturbances) - Magnetospheric physics (Solar wind interactions with unmagnetized bodies)

\section{Introduction}

When the solar wind encounters Mars it is deflected around the planet by forces, which arise in the interactions among the solar wind, the ionosphere, the neutral atmosphere and the planet's intrinsic magnetic field (crustal magnetic field, perhaps by a magnetic field induced in the ionosphere). Over most of Mars it is the solar wind interaction with the neutral atmosphere that is the important process. The neutral atmosphere above the ionopause is ionised and carried away by the solar wind, slowing the solar wind down and deflecting it in the process (e.g. Luhmann and Bauer, 1992). In this paper we examine experimental observations of electron density profiles in the ionosphere to determine if the electron densities are affected by the solar wind. Measurements made by Mars Global Surveyor (MGS) provide altitude profiles of electron density with sufficient time resolution, as well as time duration, to allow such an examination.

The distribution of electrons in the Martian ionosphere is influenced by many factors: the solar zenith angle (Zhang et al., 1990), solar radiation (Stewart and Hanson, 1982), topography (Bougher et al., 2001; Wang and Nielsen, 2004), season (Bougher et al., 1999), dust storms (Wang and Nielsen, 2003a), and solar cycle (Hantsch and Bauer, 1990). It has also been suggested that the altitude of the solar wind interaction region suffered violent influence from the solar wind (Mitchell et al., 2001). In this paper we look for signatures of the solar wind variations in the ionosphere below the interaction region.

\section{Observations}

The data set analysed here is Martian ionosphere electron density profiles obtained in December 2000 and January 2001 by the radio occultation experiment on board MGS (Hinson et al., 2003). There are several advantages of this data set compare to all previous measurements: it covers nearly two solar rotation periods; the time resolution is $2 \mathrm{~h}$ (except for missing data); all the observations were made at nearly a constant local time (2.76-3.08 LT) with slightly varying solar zenith angle $\left(82.2-75.3^{\circ}\right)$ in a rather small latitudinal region $\left(67.5-77.6^{\circ} \mathrm{N}\right)$. Thus, daily-running averages can be regarded as measurements made at nearly the same local time and at roughly the same location on sequential days. Moreover, all of the measurements are made in the area where neither noticeable crustal magnetic field nor significant topographical variation exists, so the data will not be influenced by these parameters. During this time period no violent activity such as dust storms, which could bias the study, has been reported. Any effects related to the longitude (e.g. Topographic effects, Bougher et al., 2000; Wang and Nielsen, 2003b) are easily removed by applying a running average to the data, and correction for the solar zenith angle follows the well-known methods (e.g. Zhang et al., 1990). Therefore, the data set is well suited for studies of temporal variations in the ionosphere caused by the solar wind. 


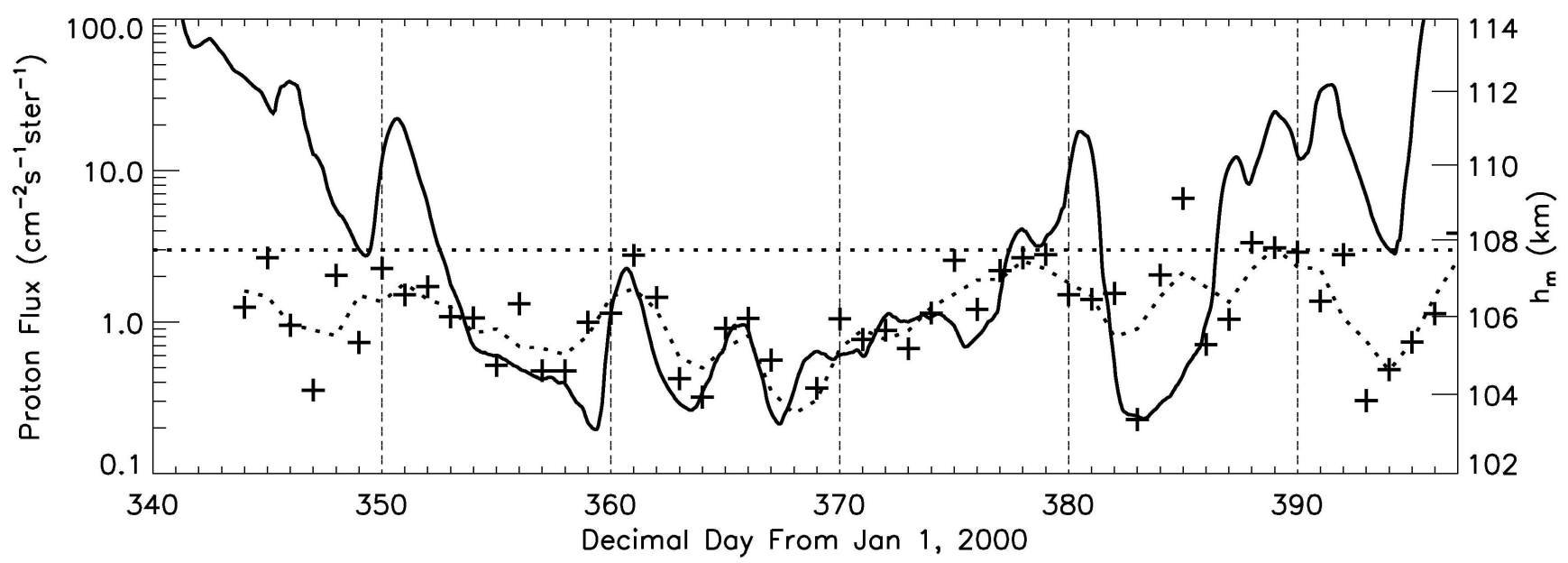

Fig. 1. The flux of protons with energy of 761 to $1220 \mathrm{keV}$ in the solar wind (solid curve) and the corrected altitude of the ionospheric electron density maximum (“+”) are plotted against the decimal day from 1 January 2000. The proton flux is obtained by ACE at the Earth's orbit and the time is shifted 7 days due to the geometry of the Sun-Earth-Mars configuration (see text for details). The dotted curve is the 3-point running average of the "+"s.

The maximum electron density and the electron density scale height above the density maximum have been found to relate to solar radiation, indicated by F10.7, as has been reported before in the literature (Stewart and Hanson, 1982; Bauer and Hantsch, 1989). However, here it is shown for the first time, that the altitude of the maximum electron density, $\mathrm{h}_{m}$ is influenced by the solar wind.

The solar wind data were measured by the Electron, Proton, and Alpha Monitor (EPAM) on board the Advanced Composition Explorer (ACE), which is located at a permanent vantage point 1.5 million $\mathrm{km}$ sunward of the Earth (Stone et al., 1998). During the period of observations Mars was located about $85^{\circ}$ away from the Earth in the direction of their orbital motion. Thus, a fixed point on the solar disk would need about 6 days to turn from pointing towards the Earth to pointing towards Mars. Considering an average solar wind speed of $\sim 400 \mathrm{~km}$, a further 1 to 2 days accounting for the time it typically takes for the solar wind to flow from the Earth's orbit to Mars' orbit must be included when comparing time variations at the Earth and at Mars.

The integrated flux $\left(\mathrm{F}_{p}\right)$ of solar wind protons with energy between 761 and $1220 \mathrm{keV}$ is shown in Fig. 1 (solid curve). It is possible to extrapolate the observations from the Earth's orbit to the Mars' (Schwenn, 1991), by considering a simple lag of 7 days. This lag has been introduced in Fig. 1, and is found to be sufficiently accurate for this study. Also plotted are the daily $\mathrm{h}_{m}$ ("+") and its 3-point-running average (dotted curve). It is evident that there is a positive correlation between the proton flux and the ionospheric peak electron density altitude. The proton flux is modulating the ionosphere: its increase and decrease result in the rise and fall of the ionospheric peak density altitude. Especially for the low flux case (when $\mathrm{F}_{p}$ is less than $\sim 3 \mathrm{~cm}^{-2} \mathrm{~s}^{-1}$ ster $^{-1}$, dashed line in Fig. 1), the correlation coefficient is close to 0.7. The peak altitude varies with the proton flux logarithmically (notice the logarithmic axis for the proton flux). For the high flux case (when $\mathrm{F}_{p}$ is more than $\sim 3 \mathrm{~cm}^{-2} \mathrm{~s}^{-1} \mathrm{ster}^{-1}$ ), the ionospheric peak altitude tends to saturate near $106.5 \mathrm{~km}$, and becomes independent of the magnitude of the proton flux.

This can be depicted more clearly by a scatter plot. In Fig. $2 \mathrm{a}, \ln \left(\mathrm{F}_{p}\right)$ is plotted against the 3-point running average values of $h_{m}$. The best linear fit is determined separately for the low and high fluxes. The fit (solid line) for the low flux case is given by $\mathrm{h}_{m}=106+0.82 \ln \left(\mathrm{F}_{p}\right)$; that only four data points are outside the \pm 1 -sigma range (shown by dashed lines parallel to the solid line) suggests that $\mathrm{h}_{m}$ is strongly dependent on $\mathrm{F}_{p}$. For the high flux case, the best-fit is a nearly horizontal line (the slope is only -0.005), suggesting that the increase in the mean ionospheric peak altitude becomes saturated with the increase in the solar wind proton flux. The maximum electron density altitude has also been related to the solar radio flux F10.7 (in Fig. 2b), and no correlation between the parameters can be discerned.

\section{Discussion}

It has been shown before that the solar radiation strongly controls some key parameters of the Martian ionosphere. It has also been shown that the solar radiation has no noticeable effects on the ionospheric peak altitude, $\mathrm{h}_{m}$. Here $\mathrm{h}_{m}$ is for the first time reported to be modulated by solar wind proton fluxes. The solar radiation control of the Martian ionosphere is much stronger than the control exerted by the solar protons. In order to account for the solar wind control of the ionosphere, we will therefore assume that the parameter influenced by the proton fluxes is not affected by the solar radiation (because otherwise the radiation effects would swamp the proton flux effects).

It is first noticed that dynamic processes in the Martian ionosphere itself can only extend down to an altitude of about 

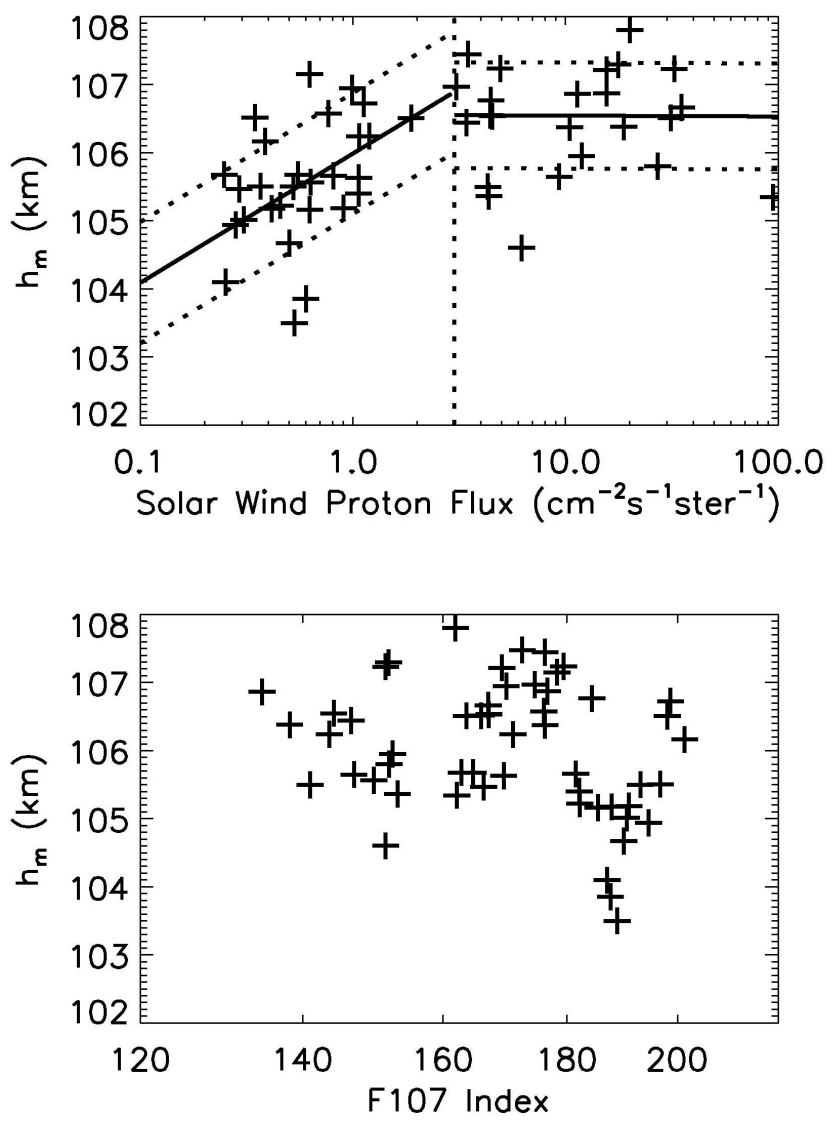

Fig. 2. The corrected altitude of the ionospheric electron density maximum versus the energetic proton flux in the solar wind (panel a) and the solar radiation F107 index (panel b). The solar wind proton flux control of the ionospheric peak altitude is different for low and high fluxes (see text for details). The solid lines in (a) is the best fit for the scattered points and the dotted lines parallel to the solid curves show \pm 1 -sigma deviations. No correlation is found between the peak altitude and F10.7 Index.

$10 \mathrm{~km}$ above the electron density maximum, $\mathrm{h}_{m}$, (Zhang et al., 1990; Wang and Nielsen, 2003b). Therefore, dynamic processes are unlikely to influence $\mathrm{h}_{m}$.

The fact that the ionospheric peak altitude is positively correlated with the solar wind energetic proton flux suggests that a possible explanation for the observed results is that the upper neutral atmosphere is ionised and heated by precipitation of the protons. Solar wind particle's deposit energy in the Martian atmosphere has been demonstrated by several authors (e.g. Kallio and Janhunen, 2001; Haider et al., 2002). Heating of the neutral atmosphere will - other parameters being constant - result in an increase in the neutral gas temperature. It has been shown before by modelling that an increase in the neutral temperature below the ionospheric peak density results in an increase in the altitude of the electron density maximum, without a noticeable change in the density of the ionospheric peak (Wang and Nielsen, 2003a). This process is very efficient, i.e. even a small temperature increase leads to a significant increase in the altitude of the maximum den-

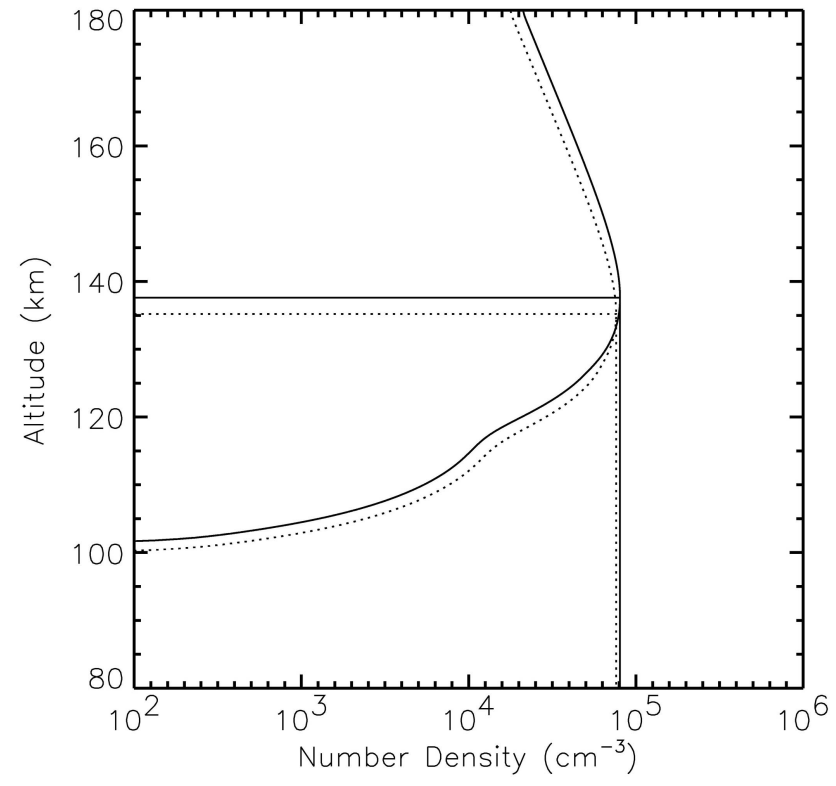

Fig. 3. The Martian ionosphere affected by the solar wind. When a $2 \mathrm{~K}$ increase in the neutral temperature at $120 \mathrm{~km}$ (and decreasing to zero at the ionopause) due to heating by solar protons is considered, then the undisturbed ionosphere (the dashed curve) changes to the ionosphere given by the solid curve. The solar zenith angle is $80^{\circ}$. The horizontal and vertical straight lines indicate the altitude and the density of the ionospheric peak in either case. Note that the increase of neutral temperature raises the peak altitude by $\sim 2.5 \mathrm{~km}$.

sity. Using a temperature dependent photochemistry model for the Martian ionosphere (Wang and Nielsen, 2003a), we find, that at a solar zenith angle of $80^{\circ}$, a $2 \mathrm{~K}$ change of the neutral temperature at $120 \mathrm{~km}$ altitude results in a $2 \sim 3 \mathrm{~km}$ increase of $\mathrm{h}_{m}$, The day-to-day variation of $\mathrm{h}_{m}$ is generally $1 \sim 2 \mathrm{~km}$. Thus, a variation of $\sim 1 \mathrm{~K}$ in the neutral air temperature below the density peak would be sufficient to account for the observations. Figure 3 shows the displacement of the ionosphere to a higher altitude, and also illustrates the near constancy of the maximum density, associated with a small increase in neutral temperature.

The strongest heating source in the upper atmosphere is the solar EUV heating (indicated by F10.7), whose variation can significantly change the neutral temperature above the ionospheric peak (Bougher et al., 2000). However, the modelling has also shown that an increase of the neutral temperature above the ionospheric peak can only change the ionospheric peak density but not its altitude. This is the reason why the solar EUV radiation can strongly affect the ionospheric peak density but not its altitude (e.g. Stewart and Hanson, 1982).

Heating of the neutral gases due to precipitation is about one order of magnitude less than that due to the solar EUV radiation (Kallio and Janhunen, 2001) and the atmospheric heating is therefore generally dominated by the EUV heating. However, there is an altitude difference in the heating rates between the two sources. The altitude of the heating 
rate peak due to precipitation of protons does not have a notable solar zenith angle (SZA) dependence, and is always centred around $120 \mathrm{~km}$; furthermore, the heating persists at night (Kallio and Janhunen, 2001). The altitude of the heating peak due to solar EUV radiation (which is the same as the altitude of the electron density maximum) increases with SZA and the heating disappears at night (Zhang et al., 1990). At the mean solar zenith angle $\left(\sim 80^{\circ}\right)$ of the data set used here the peak altitude of the precipitation heating $(\sim 120 \mathrm{~km})$ is about $20 \mathrm{~km}$ lower than that of the solar EUV heating or the ionospheric density peak $(\sim 140 \mathrm{~km})$. This low altitude heating by the precipitating protons can play a significant role for the altitude distribution of the ionospheric electrons. It is the heating at relative low altitudes that causes the increase in the altitude of the electron density maximum.

According to Kallio and Janhunen (2001), at a solar zenith angle of $75^{\circ}$, the total energy absorption rate by the neutral atmosphere from the precipitation particles from 120 to $140 \mathrm{~km}$ can be derived as $\sim 5 \times 10^{-10} \mathrm{~J} / \mathrm{cm}^{2}$ s and $\sim 24 \%$ of them are associated with ionization. Given a heating efficiency of $\sim 0.2$ (Fox and Dalgarno, 1979) and typical neutral atmosphere densities at this altitude range (e.g. Wang and Nielsen, 2003a), it can be concluded that the mean heating rate in this range is about $\sim 2 \times 10^{-5} \mathrm{~K} / \mathrm{s}$, or $\sim 1.5 \mathrm{~K} /$ day. It should be noted that this estimation is under the assumption that the original distribution function of the solar wind proton is Maxwellian (Kallio and Janhunen, 2001). However, the distribution, as a rule, deviates strongly from Maxwellian and has a pronounced proton high-energy tail which contributes a significant portion to the total particle energy (e.g. Marsch, 1991). Moreover, as pointed out above, the ionospheric peak altitude is correlated mostly to the high-energy particle flux. It implies that the heating rate derived above is underestimated. From this we find $\sim 1 \mathrm{~K}$ is a realistic estimate for the mean temperature increase in the neutral atmosphere in the focused area. And modeling shows that this $\sim 1 \mathrm{~K}$ will result in an $1 \sim 2 \mathrm{~km}$ rise in the ionospheric peak in altitude, which is also the typical day-to-day variation of the observed $\mathrm{h}_{m}$.

Since the altitude difference between the maximums of the precipitation heating and the ionospheric electron density decreases with the decrease in SZA, it can be argued that the solar wind modulation on the Martian ionosphere is more prominent at high SZA than at low SZA.

The saturation of the density maximum altitude that we have observed for large solar wind proton fluxes comes about because the upper atmosphere prevents the neutral temperature from keeping increasing for increasing input energy, the neutral temperature must level off. It is also to be noted, that before the solar wind protons precipitate into the ionosphere, they penetrate both the bow shock and the magnetic boundary above the ionosphere and their energy distribution is strongly distorted (Kallio and Janhunen, 2001).

\section{Conclusion}

Experimental evidence has been presented that the height of the electron density in the Martian ionosphere is influenced by the energetic proton flux in the solar wind, in such a way that the altitude increases when the flux increases. It has been suggested that this may be caused by a small increase in the neutral gas temperature (of the order of $1 \mathrm{~K}$ ) below the ionospheric peak electron density. It is suggested that the source of the heating is solar wind charged particles precipitating into the Martian atmosphere. The associated energy deposit may be sufficient to increase the neutral gas temperature by $\sim 1 \mathrm{~K}$, which is a heating compatible with the observed increase in the altitude of the electron density maximum. It is also argued that the solar wind control on the Martian ionosphere is more prominent at high solar zenith angle than at low solar zenith angle.

Acknowledgements. We thank the MGS Radio Science Team for making their ionospheric profile data available on a public website. We also thank that the ACE EPAM instrument team and the ACE Science Center for providing the ACE data. This research was funded by the Bundesministerium für Bildung und Forschung through the Deutsche Zentrum für Luft- and Raumfahrt e.V. (DLR) grant \# 50 QM 0004. J.-S. Wang is also partly supported by National Natural Science Foundation of China (No. 40374058, 40134020).

Topical Editor M. Lester thanks H. Rishbeth for his help in evaluating this paper.

\section{References}

Bauer, S. and Hantsch, M.: Solar cycle variation of the upper atmosphere of Mars, Geophys. Res. Lett., 16, 373-376, 1989.

Bougher, S. W., Engel, S., Roble, R. G., and Foster, B.: Comparative terrestrial planet thermospheres: 2 . Solar cycle variation of global structure and winds at equinox, J. Geophys. Res., 104, 16591-16611, 1999.

Bougher, S. W., Engel, S., Hinson, D. P., and Forbes, J. M.: Mars global surveyor radio science electron density profiles: Neutral atmosphere implications, Geophys. Res. Lett., 28, 3091-3094, 2001.

Bougher, S. W., Engel, S., Roble, R. G., and Foster, B.: Comparative terrestrial planet thermospheres: 3 . Solar cycle variation of global structure and winds at solstices, J. Geophys. Res., 105, 17 669-17 689, 2000.

Fox, L. J. and Dalgarno, A.: Ionization, luminosity, and heating of the upper atmosphere of Mars, J. Geophys. Res., 84, 7315-7333, 1979.

Haider, S. A., Seth, S. P., Kallio, E., and Oyama, K. I.: Solar EUV and electron-proton-hydrogen atom-produced ionosphere on Mars: Comparative studies of particle fluxes and ion production rates due to different processes, Icarus, 159, 18-30, 2002.

Hantsch, M. H. and Bauer, S. J.: Solar control of the Mars ionosphere, Planet. Space Sci., 38, 539-542, 1990.

Hinson, D. P.: Public access to MGS RS standard electron density profiles, http://nova.stanford.edu/projects/mgs/eds-public. html, 2003.

Kallio, E., and Janhunen, P.: Atmospheric effects of proton precipitation in the Martian atmosphere and its connection to the 
Mars-solar wind interaction, J. Geophys. Res., 106, 5617-5634, 2001.

Luhmann, J. G. and Bauer, S. J.: Solar wind effects on atmosphere evolution at Venus and Mars, in: Venus and Mars: Atmospheres, Ionospheres, and Solar Wind Interactions, Geophys. Monogr. Ser., vol. 66, edited by Luhmann, J. G., Tatrallyay, M., and Pepin, R. O., AGU, Washington D.C., 417-430,1992.

Marsch, E.: Kinetic physics of the solar wind, in: Physics of the inner heliosphere: 2. Particles, waves and turbulence, edited by Schwenn, R. and Marsch, E., Springer-Verlag, Berlin, 45-133, 1991.

Mitchell, D. L., Lin, R. P., Mazelle, C., Rème, H., Cloutier, P. A., Acuña, M. H., Connerney, J. E. P., and Ness, N. F.: Probing Mars' crustal magnetic field and ionosphere with the MGS Electron Reflectometer, J. Geophys. Res., 106, 23 419-23 427, 2001.

Schwenn, R.: Large-scale structure of the interplanetary medium, in: Physics of the inner heliosphere: 1. Large-scale phenomena, edited by Schwenn, R. and Marsch, E., Springer-Verlag, Berlin, 99-181, 1991.
Stewart, A. I. and Hanson, W. B.: Mars upper atmosphere: mean and variations, Adv. Space Res., 2, 87-101, 1982.

Stone, E. C., Frandsen, A. M., Mewaldt, R. A., Christian, E. R., Margolies, D., Ormes, J. F., and Snow, F.: The advanced composition explorer, Space Sci. Rev., 86, 1-22, 1998.

Wang, J.-S. and Nielsen, E.: Behavior of the Martian dayside electron density peak during global dust storms, Planet. Space Sci., 51, 329-338, 2003a.

Wang, J.-S. and Nielsen, E.: Wavelike structures in the Martian topside ionosphere observed by Mars Global Surveyor, J. Geophys. Res., 108, NO. E7, 5078, doi:10.1029/2003/E002078, 2003 b.

Wang, J.-S. and Nielsen, E. : Evidence for topographic effects on the Martian ionosphere, Planet. Space Sci., in press, 2004.

Zhang, M. H. G., Luhmann, J. G., Kliore, A. J., and Kim, J.: A postpioneer venus reassessment of the Martian dayside ionosphere as observed by radio occultation methods, J. Geophys. Res., 95, 14 829-14 839, 1990. 